\title{
Establishment of a lymph node metastasis model from subcutaneous tumors of gastrointestinal stromal tumor model cells
}

\author{
KAZUFUMI SAKURAMA $^{1}$, YOSHIO NAOMOTO ${ }^{1}$, TOSHIAKI OHARA $^{1}$, \\ NOBUYUKI WATANABE ${ }^{1}$, MUNENORI TAKAOKA ${ }^{1}$, HITOSHI NAGATSUKA ${ }^{2}$, \\ YASUKO TOMONO $^{3}$, TORU TANIDA ${ }^{1}$, KAZUHIRO NOMA $^{1}$, SHUNSUKE TANABE ${ }^{1}$, \\ YASUHIRO FUJIWARA ${ }^{1}$, TAKAYUKI MOTOKI ${ }^{1}$, YASUHIRO SHIRAKAWA ${ }^{1}$, \\ TOMOKI YAMATSUII ${ }^{1}$, SEIICHI HIROTA ${ }^{4}$, TAKAHIRO TAGUCHI ${ }^{5}$ and NORIAKI TANAKA ${ }^{1}$ \\ ${ }^{1}$ Departments of Gastroenterological Surgery, Transplant and Surgical Oncology and ${ }^{2}$ Oral Pathology and Medicine, \\ Graduate School of Medicine and Dentistry, Okayama University; ${ }^{3}$ Shigei Medical Research Institute, Okayama; \\ ${ }^{4}$ Department of Surgical Pathology, Hyogo College of Medicine, Nishinomiya, Hyogo; ${ }^{5}$ Department of Human \\ Health and Medical Science, Graduate School of Kuroshio Science, Kochi University, Nankoku, Kochi, Japan
}

Received September 19, 2008; Accepted November 11, 2008

DOI: 10.3892/or_00000237

\begin{abstract}
There is currently no suitable animal model of metastasis using cultured human gastrointestinal stromal tumor cells even though the molecular mechanisms of c-KITmediated progression and metastasis should be clarified. $\mathrm{Ba} / \mathrm{F} 3$ murine lymphocyte cells transduced with mutant c-KIT have been utilized to analyze some molecular mechanisms related to a constitutively activated c-KIT signaling and to assess the efficacy of molecular-targeted inhibitors. Using this cellular system, we coincidentally discovered the development of axillary and inguinal lymph node swelling three weeks after subcutaneous injection of $\mathrm{Ba} / \mathrm{F} 3$ cells with c-KIT mutation into nude mice. Mutation-specific PCR detected c-KIT mutation in the swollen lymph nodes but not in unmetastasized normal lymph nodes, indicating that the lymph nodes contain tumor cells which should come from a primary subcutaneous tumor. Microscopic observation revealed tumor cells infiltrating through lymphatic follicles with Ki-67-positive staining to distinguish them from lymphocytes. The significance of this model is helpful to understand the molecular mechanisms of c-KIT-mediated metastasis and is useful for assessments of molecular therapeutics and in vivo imaging.
\end{abstract}

Correspondence to: Dr Munenori Takaoka, Department of Gastroenterological Surgery, Transplant and Surgical Oncology, Okayama University Graduate School of Medicine, Dentistry and Pharmaceutical Sciences, 2-5-1, Shikata-cho, Okayama 700-8558, Japan

E-mail: munie41@hotmail.com

Key words: c-KIT mutation, lymph node, metastasis, gastrointestinal stromal tumor

\section{Introduction}

Human gastrointestinal stromal tumors (GISTs), which are mesenchymal tumors of the gastrointestinal tract, are believed to originate from a neoplastic transformation of interstitial cells of Cajal and often express gene mutation to c-KIT (1). The mutation of c-KIT contributes to constitutive activations of itself and the downstream effectors which play an important role in cell proliferation and survival, thereby accelerating malignant progression (2). It is also reported that the KIT mutation-positive GISTs showed more frequent liver metastases and higher mortality than KIT mutation-negative GISTs (3), although the detailed mechanism of c-KIT-mediated cell migration and metastasis has not been characterized. Molecular-based research on GIST has been facing some difficulties to be explored, partially because at the moment, there is no study regarding any suitable animal models with peritoneal dissemination or liver metastasis using cultured human GIST cells, giving the impression that it is difficult to establish a metastasis model of GIST.

$\mathrm{Ba} / \mathrm{F} 3$ murine lymphocyte-derived cell lines, which can only grow under the existence of IL-3, proliferate independent of IL-3 by stably transducing mutant c-KIT genes that are often found in clinical GIST cases (4-6). These derivatives have been utilized to analyze some molecular mechanisms related to a constitutively activated c-KIT signaling (4-8) and to assess the efficacy of molecular-targeted inhibitors such as Imatinib and Sorafenib $(9,10)$. Using this cellular system, it has been reported that $\mathrm{Ba} / \mathrm{F} 3$ cells with c-KIT mutation can form a subcutaneous tumor in nude mice $(6,9)$ and have been utilized in some investigations to help understand the molecular aspects of c-KIT mutation and its relationship to tumor progression $(4,6,9)$. Herein we report that we coincidentally discovered the development of axillary and inguinal lymph node swelling three weeks after subcutaneous injection of 
$\mathrm{Ba} / \mathrm{F} 3$ cells with c-KIT mutation into nude mice. Although it is rare for human GIST to cause lymph node metastasis, our findings can provide a certain advantage for further investigations in GIST research and this model could help us understand the molecular mechanisms of metastasis through activated c-KIT signaling.

\section{Materials and methods}

Cell lines and cultures. $\mathrm{Ba} / \mathrm{F} 3$ derivatives, stable transfectants with mutant c-KITs in original murine lymphocytes $(\mathrm{Ba} / \mathrm{F} 3)$, were generous gifts by Dr Seiichi Hirota (Hyogo College of Medicine, Nishinomiya, Japan) and were used in this study. The three types of c-KIT mutation used in this study are as described previously (4-7). Briefly, KIT ${ }^{\text {del559-560 }}$ represents an in-frame deletion of 6 bps encoding Val-559-Val-560 in the juxtamenbrane domain, KIT ${ }^{642 G l u}$ is a Lys-to-Glu substitution at codon 642 in the tyrosine kinase (TK) I domain and KIT ${ }^{820 T y r}$ is an Asp-to-Tyr substitution at codon 820 in the TK II domain. These cell lines were cultured in $\alpha$ Modified Eagle Medium ( $\alpha$ MEM) (MP Biomedicals, LLC, Solon, OH) supplemented with $10 \%$ fetal bovine serum (FBS) (Invitrogen Co., Carlsbad, CA) and maintained in culture at $37^{\circ} \mathrm{C}$ in humidified air with $5 \% \mathrm{CO}_{2}$.

Animal experiments. All mouse experiments were carried out in accordance with the standards and guidelines of the Department of Animal Resources at Okayama University and followed principles of laboratory animal care $(\mathrm{NIH}$ publication No. 85-23, revised 1985). Eight week-old female BALB/ cAjcl-nu/nu mice derived from C.B-17/Icr (Clea, Tokyo, Japan) were used in this study. All mice were maintained under pathogen-free conditions. When the cells were propagated enough, they were collected as a pellet by a five minute-centrifugation at $3000 \mathrm{rpm}$ and were resuspended with growth media to make a suspension of $5 \times 10^{6}$ cells in $100 \mu 1$. The cell suspension was mixed with Matrigel (BD Biosciences, San Jose, CA) at a 1:1 ratio and $200 \mu$ l of the final mixture was injected subcutaneously near the iliac bone in the right back of an anesthesized mouse. Three weeks later, the mice were sacrificed for observation and resection of tissues.

Reverse transcription-polymerase chain reaction (RT-PCR). Total RNA was prepared by RNAzol (Tel-Test, Inc., USA) from tissues resected from the mice. An input of $1 \mu \mathrm{g}$ total RNA was reverse transcribed with a PrimeScript 1st strand cDNA synthesis kit according to the manufacturer's instructions (Takara Biotechnology Co. Ltd., Japan).

Subsequent PCRs were carried out to specifically detect the del559-560 type of c-KIT mutation using KOD plus DNA polymerase (Toyobo Co. Ltd., Osaka, Japan) and the following set of primers; forward: 5'-gta caa tgg aag gag gag ata aat gg-3', reverse: 5'-tct tgc gga tct cct ctt gt-3'. For c-KIT cDNA amplification, an initial denaturation at $94^{\circ} \mathrm{C}$ for 10 min was followed by 35 cycles of a denaturation step at $94^{\circ} \mathrm{C}$ for $30 \mathrm{sec}$, an annealing step at $60^{\circ} \mathrm{C}$ for $45 \mathrm{sec}$ and an extension step at $68^{\circ} \mathrm{C}$ for $1 \mathrm{~min}$. The amplified 558-bp products were electrophoresed in $1 \%$ agarose gel for visualization in a UV-transilluminator.
Table I. Incidence of lymph node swelling in Ba/F3 subcutaneous xenograft.

\begin{tabular}{lccc}
\hline \multirow{2}{*}{ Cell line } & Mutation site & \multicolumn{2}{c}{ Sites of lymph nodes } \\
\cline { 3 - 4 } & & Axillary & Inguinal \\
\hline \multirow{2}{*}{ KIT $^{\text {del559-560 }}$} & Juxtamembrane (exon 11) & $3 / 3$ & $3 / 3$ \\
KIT $^{642 \text { Glu }}$ & Tyrosine kinase I (exon 13) & $3 / 3$ & $3 / 3$ \\
KIT $^{820 T y r}$ & Tyrosine kinase II (exon 17) & $3 / 3$ & $3 / 3$ \\
Control & - & $0 / 3$ & $0 / 3$ \\
\hline
\end{tabular}

Immunohistochemistry. Resected tissues were fixed with $10 \%$ formalin and paraffinized for sectioning. Embedded 5- $\mu \mathrm{m}$ sections on silanized slides were subjected to immunohistochemical staining following a procedure described previously (11). Briefly, after endogenous peroxidase activity was blocked using $3.0 \% \mathrm{H}_{2} \mathrm{O}_{2}$ in methanol for $15 \mathrm{~min}$, antigen retrieval was performed by heating in $10 \mathrm{~mm}$ citrate buffer solution ( $\mathrm{pH}$ 6.0), followed by blocking of nonspecific reactivity with rabbit serum. Overnight-incubation with a primary antibody at $4^{\circ} \mathrm{C}$ and subsequent labelings with a biotinylated anti-primary antibody and streptavidin peroxidase were performed before visualization. Color development was achieved using $\mathrm{DAB} / \mathrm{H}_{2} \mathrm{O}_{2}$ solution (Histofine DAB substrate kit; Nichirei) and Mayer's hematoxylin as counterstaining. Some sections were subjected to normal serum blocking and omission of the primary antibody as a negative control. The primary antibody used in this study was anti-Ki-67 (clone MIB-1) monoclonal antibody (Dako Cytomation A/S, Copenhagen, Denmark).

\section{Results and Discussion}

Macroscopic views of the lymph node swelling on the 21st day after subcutaneous injection of tumor cells. When $\mathrm{Ba} / \mathrm{F} 3$ derivatives were subcutaneously inoculated into nude mice, all of them formed tumors on the inoculated sites. While the subcutaneous tumors were growing, macroscopic swelling of the lymph nodes at the axillary and inguinal lesions was eventually recognized 21 days from the date of cell injection (Fig. 1). Surgical observation by opening the skin of the mice showed an obvious existence of lymph node swelling in those areas with a certain distance from the original tumor on the back. Of note, the axillary lymph nodes were recognized by their milky white tone, whereas the inguinal lymph nodes were colored a reddish brown. We confirmed that all of tumorbearing mice revealed swollen lymph nodes both in axillary region and in inguinal region and each region contained 1 or 2 swollen masses (Table I). This observation tempted us to speculate that the swelling of the lymph nodes may have been due to metastasis from the subcutaneous tumors.

Microscopic observation of primary subcutaneous tumors. When hematoxylin and eosin staining was applied to paraffin sections of resected tissues, each of the subcutaneous tumors was filled with tumor cells, which exhibited a large N/C ratio regardless of c-KIT mutation type (Fig. 2). Microscopic 
a

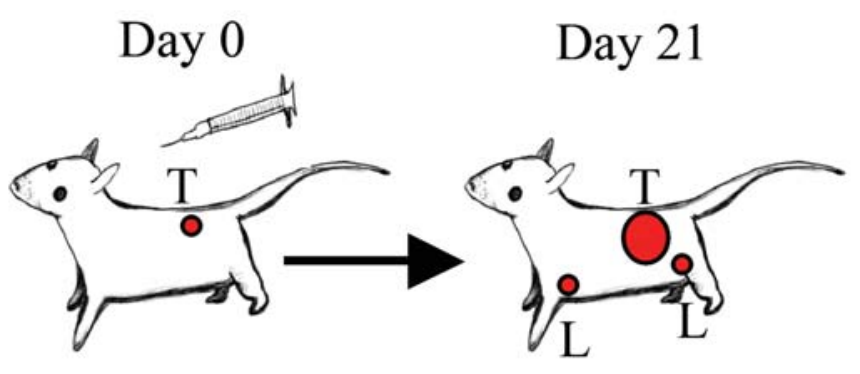

b

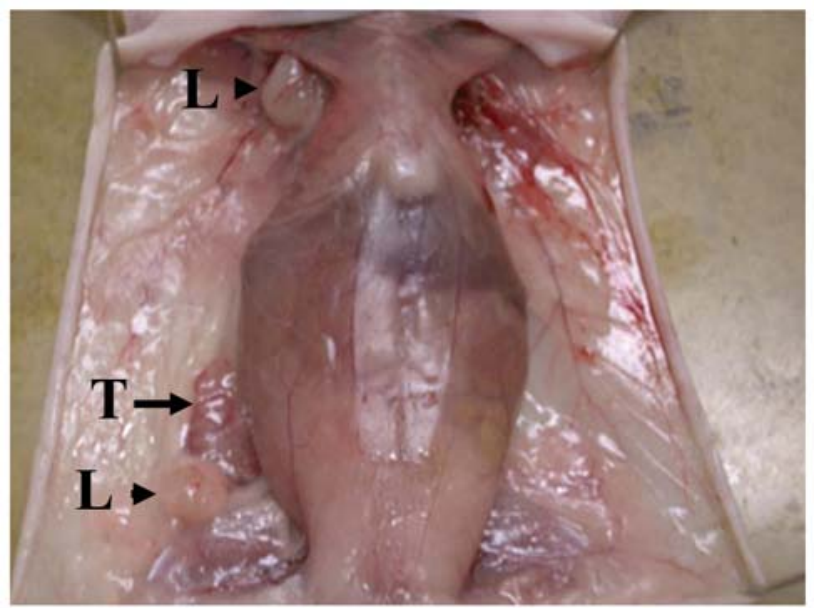

Figure 1. Representative image of subcutaneous tumor formation and subsequent swelling of lymph nodes. The schematic procedure of this model is shown in panel (a). Firstly, Ba/F3 cells were inoculated to make subcutaneous tumors on the back of nude mice. The axillary and inguinal lesions were eventually swollen 21 days after the inoculation. $\mathrm{T}$ and $\mathrm{L}$ in the panel stand for subcutaneous tumor and swollen lymph nodes, respectively. (b) Macroscopic view of the animal model is shown. A primary subcutaneous tumor $(\mathrm{T})$ and swollen lymph nodes at the axillary and inguinal lesions (L) were recognized.

observation of the swollen lymph nodes indicated that tumor cells, which looked the same as the ones in the primary subcutaneous tumors, occupied most of the swollen lymph nodes and that the remaining lymphocytes maintained a margin of the mass (Fig. 3). This finding suggested that the swelling of these lymph nodes may have been caused by the infiltration of tumor cells from the original subcutaneous tumors.

Detection of c-KIT mutation in the swollen lymph nodes as a direct proof of lymph nodal metastasis. To get a direct evidence that the swollen lymph nodes were occupied with metastasized tumor cells, RT-PCR was carried out to specifically detect c-KIT mutation which should be originated from the primary subcutaneous tumors but not from any other sites nor from de novo neoplasms. Fig. 4 exhibited an apparent detection of deletion-type mutation of c-KIT in a swollen lymph node that was taken out of a mouse bearing a subcutaneous tumor of $\mathrm{Ba} / \mathrm{F} 3-\mathrm{c}-\mathrm{KIT} \mathrm{T}^{\mathrm{del} 559-560}$ cells. This result supported our observation that $\mathrm{Ba} / \mathrm{F} 3$ derivatives with mutant $\mathrm{c}-\mathrm{KIT}$ indeed infiltrated into the axillary and inguinal lymph nodes and that this locomotion was a metastasis from the original subcutaneous tumors.
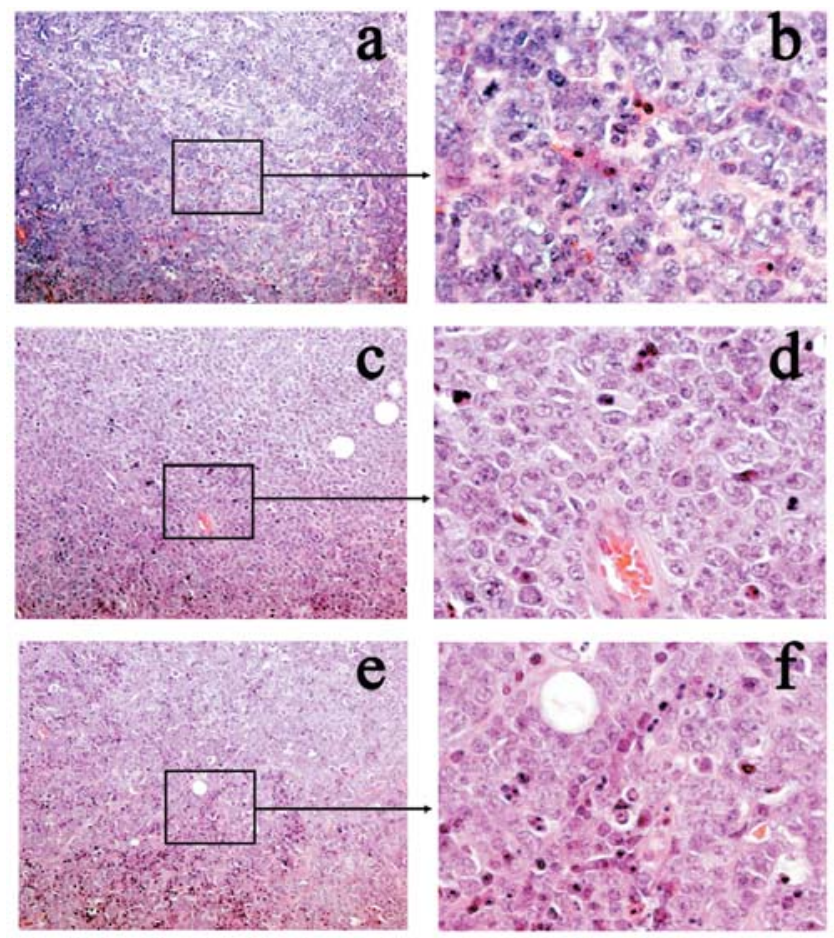

$\mathrm{x} 100$

$\mathrm{x} 400$

Figure 2. Microscopic characteristics of subcutaneous tumors of Ba/F3 cells with mutant c-KIT. Hematoxylin and eosin staining revealed that each tumor ( $\mathrm{a}$ and b: KITdel559-560, $\mathrm{c}$ and d: KIT ${ }^{642 \mathrm{Glu}}$ and e and f: KIT ${ }^{820 \mathrm{Tyr}}$ ) contained proliferating cells with a large $\mathrm{N} / \mathrm{C}$ ratio. Original magnifications: a, c and e: $x 100, b, d$, and f: $x 400$.

Indirect proof of tumor cells in swollen lymph nodes with close attention to the difference of growth abilities. We further assessed the ability of proliferation in metastasized tumor cells in swollen lymph nodes to support our observation of regional lymph node metastasis. Immunohistochemical staining for Ki-67, a marker for active proliferation, was used to distinguish tumor cells, which have a potent ability to grow, from lymphocytes, because lymphocytes should be terminally differentiated and are usually quiescent in their growth status. As shown in Fig. 5, more Ki-67-positive staining was detected in the swollen lymph nodes than in normal lymph nodes and the positive staining was observed in the swollen lymph nodes corresponding to tumor cells with a large N/C ratio. Statistical analysis revealed that the positive-stained cells were significantly more in swollen lymph nodes than in normal lymph nodes (Fig. 5e). On the other hand, there was no positive staining in the lymphocytes adjacent to the tumor cells (Fig. 5a and b). A subset of positive-stained cells were observed at the center of normal lymph nodes though, we assumed that they might be metrocytes such as stem cells (Fig. 5c and d).

The significance of the establishment of a lymph nodal metastasis model. To our knowledge, there is currently no established model of metastasis with GIST cells, although GIST rarely metastasizes to lymph nodes in clinical practice. Due to the extreme difficulty of establishing GIST tissue-origin culture cell lines, Ba/F3 cellular system has been utilized to analyze some molecular mechanisms related to constitutively 

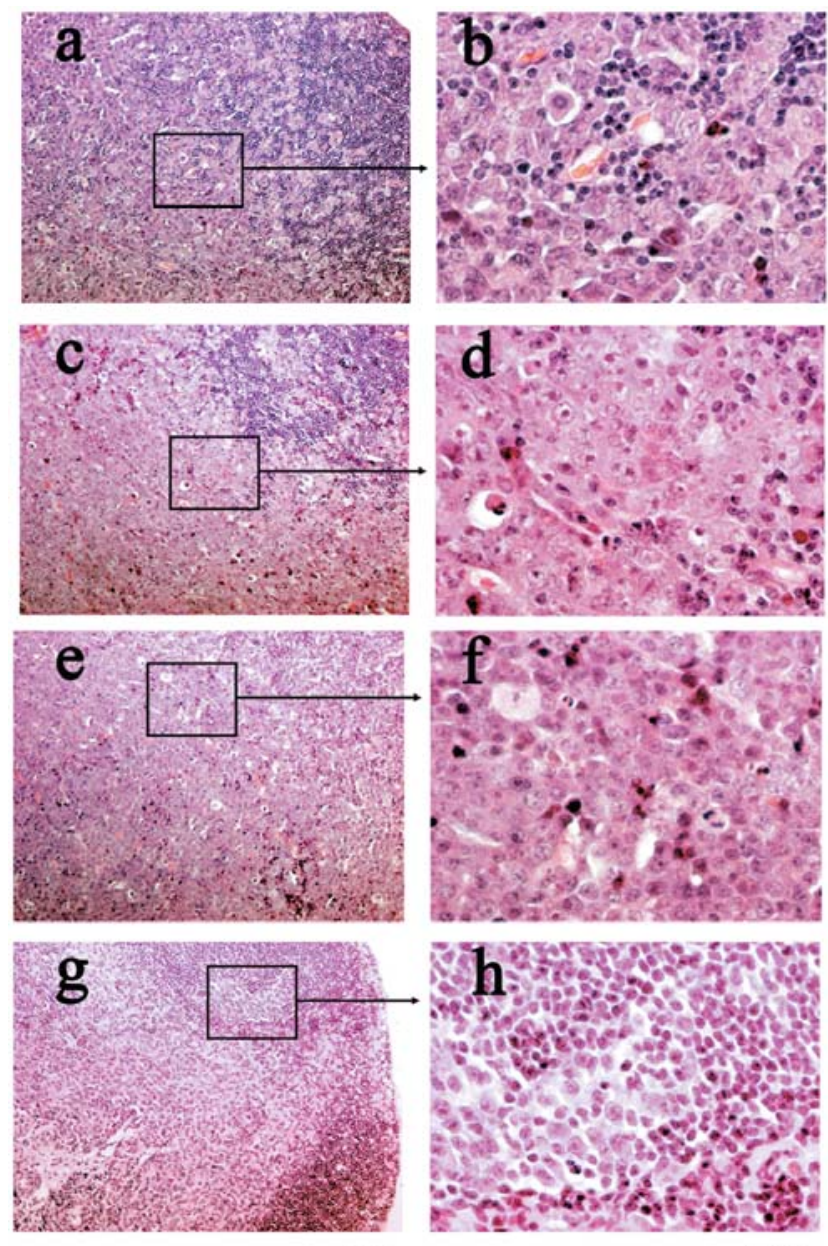

$\mathrm{x} 100$
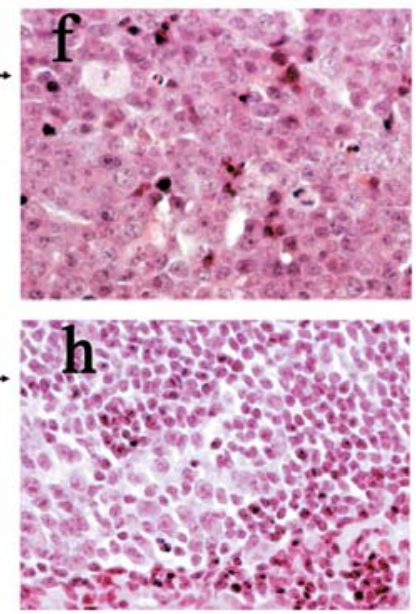

$\mathrm{x} 400$

Figure 3. Infiltrating tumor cells in swollen lymph nodes of subcutaneous tumor-bearing mice. Hematoxylin and eosin staining revealed that the swollen lymph nodes were infiltrated by tumor cells ( $\mathrm{a}$ and $\mathrm{b}$ : KIT ${ }^{\mathrm{del} 559-560}$, c and d: KIT ${ }^{642 G l u}$, e and f: KIT $^{820 T y r}$, while only lymphatic follicles were recognized in normal lymph nodes ( $\mathrm{g}$ and $\mathrm{h}$ ). Original magnifications: a, c, e and g: x100 and b, d,f, and h: x400.

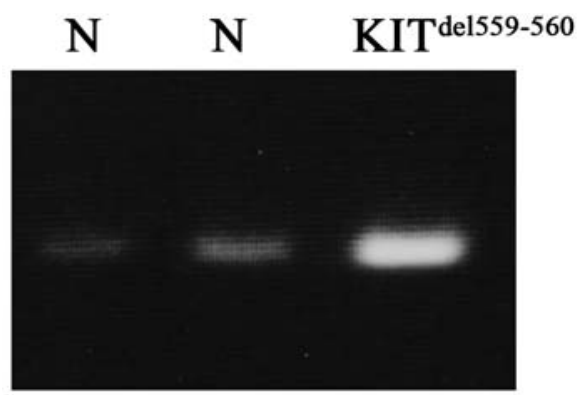

Figure 4. Mutant form of c-KIT was detected in swollen lymph nodes by mutation-specific PCR. Resected tissues were subject to a detection of tumor cells with mutant c-KIT by RT-PCR. Normal lymph nodes were taken from the control nude mice without tumors, while the swollen lymph nodes originated from the mice with subcutaneous tumors of $\mathrm{Ba} / \mathrm{F} 3$ derivatives.

active c-KIT signaling (4-8) and to assess the efficacy of molecular-targeted inhibitors $(9,10)$. In this study, we successfully established a model that $\mathrm{Ba} / \mathrm{F} 3$ cells transduced with c-KIT mutation metastasized from the original subcutaneous site to the lymph nodes. Parental $\mathrm{Ba} / \mathrm{F} 3$ cells,
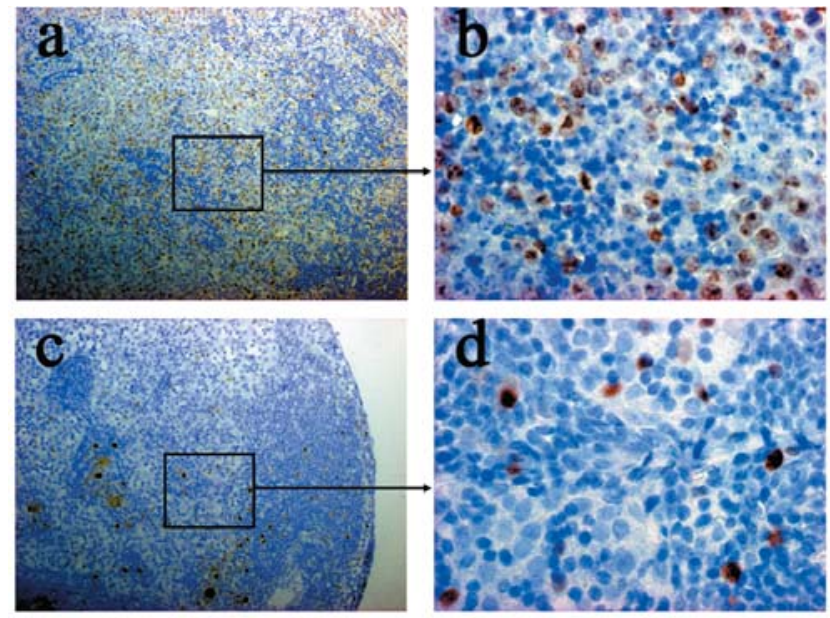

$\mathrm{x} 100$

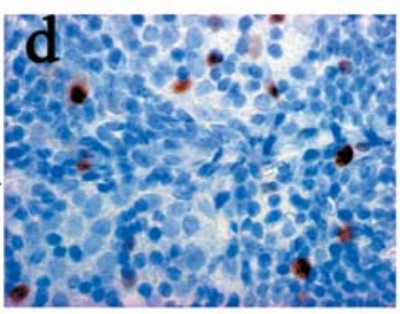

$\mathrm{x} 400$

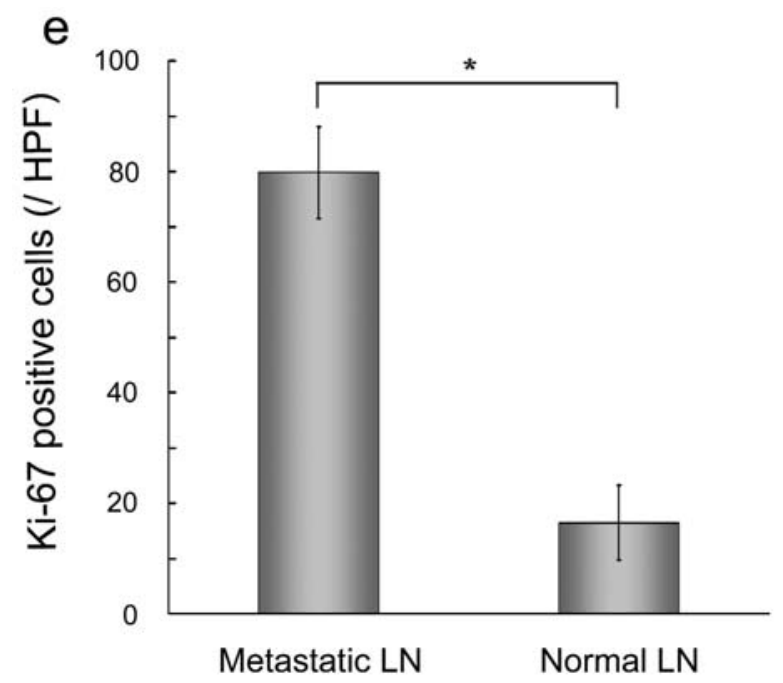

Figure 5. Immunohistochemistry for Ki-67 in swollen lymph nodes. Ki-67 staining was carried as a marker for a potent proliferating ability to distinguish the tumor cells from other lymphocytes. Ki-67-positive staining in large N/C cells was easily found in the swollen lymph nodes (a and b), whereas only a few cells stained positive for Ki-67 in the normal lymph nodes (c and d). Original magnifications: a and c: $x 100, b$ and $d: x 400$. (e) The number of Ki-67-positive-stained cells was counted in every 5 high-power field (HPF; $\mathrm{x} 400$ ) in swollen lymph nodes as well as in normal lymph nodes. Each column and error bar represents average \pm standard deviation (SD). ${ }^{*} \mathrm{P}<0.00001$.

which grow dependent of IL-3 with intact c-KIT, cannot form subcutaneous tumors $(6,9)$. On the other hand, once the cells are exogenously transduced with mutant c-KIT, the cells can grow even without IL-3 (6) and develop tumors on the backs of nude mice, followed by metastatic swelling of the lymph nodes. This suggests that a constitutive activation of c-KIT causes an increased ability of tumor growth and cell motility. The significance of this model is that it helps to understand the molecular mechanisms of c-KIT-mediated metastasis and that it may be useful to assess chemotherapeutic effects of compounds targeting c-KIT signaling, since upregulation of c-KIT is observed as a subset of malignant melanoma $(12,13)$ and small cell carcinoma of the lung (14) in addition to GISTs. It should be noted that cell growth is faster in the following order: KIT ${ }^{\text {del559-560 }}>$ KIT $^{642 \text { Glu }}>$ KITT $^{820 T y r}$ (9) and that the lymph node swelling also tends to appear according to the same 
order. Since the only difference of molecular background between those cells was the type of c-KIT mutation, the status of c-KIT mutation and its activity could be closely related with the potential of tumor progression and mortality in this metastasis model.

In conclusion, we have established a promising model that $\mathrm{Ba} / \mathrm{F} 3$ cells with mutant $\mathrm{c}-\mathrm{KIT}$ genes, which are often observed in clinical GIST cases, can be metastasized from a primary subcutaneous tumor to the axillary and inguinal lymph nodes in three weeks. We expect that this model is useful to understand the molecular mechanisms of c-KIT-mediated tumor progression and metastasis in vivo.

\section{References}

1. Heinrich MC, Rubin BP, Longley BJ, et al: Biology and genetic aspects of gastrointestinal stromal tumors: KIT activation and cytogenetic alterations. Hum Pathol 33: 484-495, 2002.

2. Vlahovic G and Crawford J: Activation of tyrosine kinases in cancer. Oncologist 8: 531-538, 2003.

3. Cho S, Kitadai Y, Yoshida S, Tanaka S, et al: Deletion of the KIT gene is associated with liver metastasis and poor prognosis in patients with gastrointestinal stromal tumor in the stomach. Int J Oncol 28: 1361-1367, 2006.

4. Hirota S, Isozaki K, Moriyama Y, et al: Gain-of-function mutations of c-kit in human gastrointestinal stromal tumors. Science 279: 577-580, 1998.
5. Nakahara M, Isozaki K, Hirota S, et al: A novel gain-of-function mutation of c-kit gene in gastrointestinal stromal tumors. Gastroenterology 115: 1090-1095, 1998.

6. Isozaki $\mathrm{K}$, Bonoit $\mathrm{T}$, Jacques $\mathrm{B}$, et al: Germline-activating mutations in the kinase domain of KIT gene in familial gastrointestinal stromal tumors. Am J Pathol 157: 1581-1585, 2000.

7. Hirota S, Nishida T, Isozaki K, et al: Familial gastrointestinal stromal tumors associated with dysphagia and novel type germline mutations of KIT gene. Gastroenterology 122: 1493-1499, 2002.

8. Debiec RM, Cools J, Dumez H, et al: Mechanisms of resistance to imatinib mesylate in gastrointestinal stromal tumors and activity of the PKC412 inhibitor against imatinib-resistant mutants. Gastroenterology 128: 270-279, 2005.

9. Chen $\mathrm{H}$, Isozaki $\mathrm{K}$, Kinoshita $\mathrm{K}$, et al: Imatinib inhibits various types of activating mutant kit found in gastrointestinal stromal tumors. Int J Cancer 105: 130-135, 2003.

10. Guo T, Agaram NP, Wong GC, et al: Sorafenib inhibits the imatinib-resistant KITT670I gatekeeper mutation in gastrointestinal stromal tumor. Clin Cancer Res 13: 4874-4881, 2007.

11. Takaoka M, Naomoto Y, Ohkawa T, et al: Heparanase expression correlates with invasion and poor prognosis in gastric cancers. Lab Invest 83: 613-622, 2003.

12. Curtin JA, Busam K, Pinkel D, et al: Somatic activation of KIT in distinct subtypes of melanoma. J Clin Oncol 24: 4340-4346, 2006.

13. Antonescu CR, Busam KJ, Francone TD, et al: L576P KIT mutation in anal melanomas correlates with KIT protein expression and is sensitive to specific kinase inhibition. Int J Cancer 121: 257-264, 2007.

14. Potti A, Moazzam N, Ramar K, et al: CD117 (c-kit) overexpression in patients with extensive-stage small-cell lung carcinoma. Ann Oncol 14: 894-897, 2003. 